\title{
A Bayesian approach for the stochastic modeling error reduction of magnetic material identification of an electromagnetic device
}

\author{
A Abdallh, G Crevecoeur and L Dupré \\ Department of Electrical Energy, Systems and Automation, Ghent University, \\ Sint-Pietersnieuwstraat 41, B-9000 Ghent, Belgium \\ E-mail: Ahmed.Abdallh@UGent.Be
}

Received 12 July 2011, in final form 22 December 2011

Published DD MM 2012

Online at stacks.iop.org/MST/23/000000

\begin{abstract}
Magnetic material properties of an electromagnetic device can be recovered by solving an inverse problem where measurements are adequately interpreted by a mathematical forward model. The accuracy of these forward models dramatically affects the accuracy of the material properties recovered by the inverse problem. The more accurate the forward model is, the more accurate recovered data are. However, the more accurate 'fine' models demand a high computational time and memory storage. Alternatively, less accurate 'coarse' models can be used with a demerit of the high expected recovery errors. This paper uses the Bayesian approximation error approach for improving the inverse problem results when coarse models are utilized. The proposed approach adapts the objective function to be minimized with the $a$ priori misfit between fine and coarse forward model responses. In this paper, two different electromagnetic devices, namely a switched reluctance motor and an EI core inductor, are used as case studies. The proposed methodology is validated on both purely numerical and real experimental results. The results show a significant reduction in the recovery error within an acceptable computational time.
\end{abstract}

Keywords: Bayesian approximation error approach, coarse and fine models, inverse problem, magnetic material identification, modeling error

Q1 (Some figures may appear in colour only in the online journal)

\section{Introduction}

Magnetic materials are indispensable components in all electromagnetic devices (EMDs) and are widely used in electrical power applications, such as rotating electrical machines. The magnetic material properties in these EMDs are classically identified by means of standard techniques. Measurements are then carried out on a separate sheet of the same material as the EMD by using an Epstein frame, single sheet tester or ring core measurements [1]. However, this requires extra samples of the electrical steel sheet of which the EMD is manufactured, which are often unattainable. Moreover, the magnetic material characteristic may be changed during the construction of the EMD, e.g. due to the introduced cutting stresses [2]. Therefore, it is convenient to characterize the magnetic properties on the specific geometry of the EMD itself.

Recently, an inverse problem has been proposed where a set of well-chosen measurements are interpreted through the use of a computer model [3]. These measurements are carried out on the geometry of the EMD itself. In practice, two major aspects can reduce the accuracy of the recovered solution when solving the inverse problem, specifically: measurement noise and inaccurate modeling [4]. Measurement noise can be eliminated or reduced to some extent by accurately performing the measurements, see [5]. On the other hand, modeling 
errors are basically originated from two main sources: the uncertain 'geometrical' model parameters and the way of modeling of the physical phenomena of the EMD. The effect of the uncertain geometrical model parameters on the solution of the inverse problem has been extensively investigated by the authors, see [6, 7]. In these references, the EMD models are assumed to be perfect, i.e. all physical phenomena are modeled, or in other words, the EMD models exactly simulate the reality. However, in practice, this assumption never happens. In this paper, we focus only on the modeling error due to the inaccurate modeling.

Generally, there are two approaches to model an EMD: analytical models and numerical models. Analytical models are computationally fast but less accurate. Numerical models can be divided into several accuracy levels depending on the degree of freedom and discretization. The two- or threedimensional finite-element (2D-FE or 3D-FE) models are commonly used with different mesh discretization levels. In general, numerical models are often more accurate than the analytical ones, but much more time consuming: the higher the degree of freedom, the more the time consumed [8].

In order to solve an inverse problem with the highest accuracy, one may incorporate the measurements with the most accurate (fine) model '3D numerical FE model' of an EMD. However, the computational time of this approach may be prohibitive. Alternatively, less accurate (coarse) models can be used with an expected higher recovery error compared to the fine models.

In this paper, we propose the use of the Bayesian approximation error approach [9] for improving the inverse problem solution when the coarse model is utilized. The proposed approach adapts the objective function to be minimized with the a priori misfit between the fine and coarse forward model responses, in which the modeling error is represented in a stochastic way.

The Bayesian approximation error approach has been successfully applied onto applications such as electrical impedance tomography (EIT) [9] and optical diffusion tomography [10]. In these applications, biomedical inverse problems are solved and different types of modeling errors are discussed: modeling errors caused by domain truncation, reduced discretization and unknown electrode contact impedances [11]; modeling errors due to unknown domain boundary [12]. It is the first time that the Bayesian approximation error approach is applied for the magnetic material characterization of an EMD. Moreover, we propose in this paper a novel criterion for selecting the best coarse model from different coarse models with varying fidelity. The proposed criterion is based on evaluating the degree of the coarseness of the coarse model that provides an a priori indication about the usefulness of the applicability of the Bayesian approximation error approach.

In order to validate the proposed methodology, purely numerical results are used for recovering the magnetic material characteristics of a switched reluctance motor (SRM). Moreover, the proposed methodology is applied for retrieving the material parameters of an EI core inductor by solving an inverse problem starting from real experimental data performed externally on this specific EMD.
The methodology is presented in section 2. Three techniques for modeling EMDs are shown in section 3 . In section 4.1, the proposed methodology is applied to a $\mathrm{SRM}$ in order to reduce the recovery error in the inverse solution resulting from inaccurate modeling. In this case study, numerical results are used, i.e. no measurements are performed. In section 4.2 , the proposed methodology is validated 'experimentally' by solving an inverse problem starting from real experimental data on an EI core inductor. In this case study, two relative coarse models, compared to the fine model, are tested. The conclusions are drawn in section 5 .

\section{Methodology}

An electromagnetic inverse problem procedure requires a large number of evaluations in forward models. Classically, for the sake of simulating as close as possible the reality, a very fine numerical model is used [13]. However, the forward model calculation and consequently the inverse problem is time demanding especially for complex geometries such as a rotating electrical machine. In order to overcome this problem, two-level methods can be used, e.g. space mapping [14]. In these two-level methods, an additional coarse model is incorporated besides the fine model so as to accelerate the inverse procedure. In fact, the two-level technique is successfully applied for magnetic material identification of a SRM [15]. Moreover, the authors presented in [8] a two-level refined direct method for electromagnetic inverse problems, in which the coarse model is based on the numerical fine model but with coarse discretizations. Although the results presented in [8] are acceptable, the implementation of these two-level techniques requires advanced computations, such as the computation of Kriging metamodels. Moreover, in the two-level methods, both fine and coarse models are solved in the iterative inverse approach. Therefore, a much easier and faster inverse scheme is needed. In this paper, we propose the use of the Bayesian approximation error for compensating the modeling error when the coarse model is used in the inverse problem instead of the fine model. In section 2.1, the modeling error is represented in a stochastic way. The traditional Bayesian and the modified Bayesian approximation error approaches are presented in sections 2.2.1 and 2.2.2, respectively.

\subsection{Stochastic representation of the modeling error}

Assume that we have two computer models of an EMD: a fine and a coarse model. The fine model is assumed to be the most accurate model, but much more time consuming, e.g. a $3 \mathrm{D}-\mathrm{FE}$ model. The coarse model is assumed to be less accurate model, but computationally fast, e.g. an analytical model. In fact, it is impossible to construct an exact computer model combining the whole physical phenomena as in the reality. However, in order to solve an inverse problem, a computer model is needed with the highest possible accuracy: the most accurate the model, the most accurate the recovery results.

In this part, we assume that the fine model is close to the reality where we assume that the modeling error in the fine 
model is negligible. However, the modeling error in the coarse model is referred to as the misfit between the fine and coarse forward model responses. We present the stochastic modeling error as follows.

The behavior of a magnetic system can be represented by a mathematical model with a set of partial differential equations. This model is parameterized by the following model parameters: the unknown parameters $\mathbf{u} \in \mathbb{R}^{p}$, i.e. the parameters to be reconstructed by the inverse problem and the precisely known parameters $\mathbf{d} \in \mathbb{R}^{r}$.

Indeed, the fine and coarse forward model responses, $\boldsymbol{\Phi}_{f} \in \mathbb{R}^{K}$ and $\boldsymbol{\Phi}_{c} \in \mathbb{R}^{K}$ respectively, depend on $\mathbf{u}$, where $K$ is the total number of observations. Since the exact value of $\mathbf{u}$ is not known, we assume $Z$ hypothetical values of the unknown model parameters, with $\widehat{\mathbf{u}}_{z}, z=1, \ldots, Z$, being a hypothetical value. These $Z$ hypothetical values are chosen in such a way that they are random and cover the domain defined by the lower and upper bounds of these parameters.

The error between the fine and coarse forward models, at each model observation $k$ and at each test value $\widehat{\mathbf{u}}_{z}$, can be represented by

$$
\begin{aligned}
e_{m, k}\left(\widehat{\mathbf{u}}_{z}\right)= & \Phi_{f, k}\left(\widehat{\mathbf{u}}_{z}\right)-\Phi_{c, k}\left(\widehat{\mathbf{u}}_{z}\right), \quad(k=1, \ldots, K), \\
& (z=1, \ldots, Z),
\end{aligned}
$$

with $K$ and $Z$ being the total number of discrete model observations and the total number of the assumed hypothetical values of the unknown model parameters, respectively. By performing $Z$ coarse and fine forward model computations, and assuming that the modeling error at each model observation $k\left(e_{m, k}\right)$ follows the normal distribution, i.e. $\left(e_{m, k} \sim\right.$ $\left.\mathcal{N}\left(\mu_{m, k}, \sigma_{m, k}^{2}\right)\right)$, one may calculate the mean modeling error and its variance, $\mu_{m, k}$ and $\sigma_{m, k}^{2}$, respectively:

$\mu_{m, k}=\frac{1}{Z} \sum_{z=1}^{Z} e_{m, k}\left(\widehat{\mathbf{u}}_{z}\right), \quad \sigma_{m, k}^{2}=\frac{1}{Z} \sum_{z=1}^{Z}\left(e_{m, k}\left(\widehat{\mathbf{u}}_{z}\right)-\mu_{m, k}\right)^{2}$.

Based on the calculated $\mu_{m, k}$ and $\sigma_{m, k}^{2}$, the approximate overall probability distribution function (PDF), at each model observation $k$, can be visualized as

$$
f\left(e_{m, k}\right)=\frac{1}{\sqrt{2 \pi \sigma_{m, k}^{2}}} \cdot \exp ^{\left(-\left(e_{m, k}-\mu_{m, k}\right)^{2} /\left(2 \sigma_{m, k}^{2}\right)\right)} .
$$

The assumption of the modeling error to be Gaussian distributed is a possible means of statistically expressing the modeling error, see [9]. The vector representations of the modeling mean error and its variance at all model observations $K$ are

$$
\begin{gathered}
\overline{\boldsymbol{\mu}}_{m}=\left[\mu_{m, 1}, \mu_{m, 2}, \ldots, \mu_{m, K}\right]^{T}, \\
\overline{\boldsymbol{\sigma}}_{m}^{2}=\left[\sigma_{m, 1}^{2}, \sigma_{m, 2}^{2}, \ldots, \sigma_{m, K}^{2}\right]^{T} .
\end{gathered}
$$

Since the modeling error is assumed uncorrelated, i.e. $e_{m, i}$ does not depend on $e_{m, j}(i, j=1, \ldots, K, i \neq j)$, the covariance matrix of the modeling error $\left(\overline{\boldsymbol{\Sigma}}_{m}^{2} \in \mathbb{R}^{K \times K}\right)$ can be written as $\overline{\boldsymbol{\Sigma}}_{m}^{2}=\operatorname{diag}\left(\sigma_{m, 1}^{2}, \sigma_{m, 2}^{2}, \ldots, \sigma_{m, K}^{2}\right)$. The mean and the covariance of the modeling error are used in the following section for the modeling error compensation.
2.1.1. Model coarseness criterion. In fact, a fine model can be simplified by several coarse models depending on the fidelity of the model. Of course, the more accurate the coarse model, the less modeling errors are introduced and vice versa. We are convinced that the Bayesian approximation error approach is helpful for reducing the modeling error to an acceptable value when the used coarse model is accurate enough to simplify the fine model. Therefore, there is a need to define a criterion to measure 'quantitatively' the coarseness degree of a model. This model coarseness criterion is implemented here for selecting a priori the best coarse model that gives acceptable results when the Bayesian approximation error approach is utilized.

The model coarseness criterion is defined based on the 95\% confidence interval. As shown above, the modeling error at each model observation $k$, i.e. $e_{m, k}$, is assumed to follow the Gaussian 'normal' distribution. Based on the mean and the standard deviation of the modeling error at each model observation $k$, i.e. $\mu_{m, k}, \sigma_{m, k}$, one may calculate the $95 \%$ confidence interval.

The modeling error (at this model observation $k$ ) is assumed to be 'True', which means that the error is small enough, if the zero error is located inside the $95 \%$ confidence interval, as shown in figure $1(a)$. However, the modeling error (at this model observation $k$ ) is assumed to be 'False', which means that the error is large, if the zero error is located outside the $95 \%$ confidence interval, as shown in figure $1(b)$. The percentage degree of the model coarseness $\eta$ is defined as the number of 'True' cases divided by the total number of the model observations $K$. The coarse model is assumed to be 'statistically' acceptable for implementing the Bayesian approach if $\eta$ exceeds a predefined threshold value, e.g. $\eta \geqslant 50 \%$.

\subsection{Bayesian approach: traditional and approximation error}

In the inverse problem framework, in order to estimate the unknown parameters $\mathbf{u}$, an inverse problem has to be solved by iteratively minimizing the quadratic residuals between the experimental observations of the magnetic system $\mathbf{W} \in \mathbb{R}^{K \times 1}$ and the modeled ones $\boldsymbol{\Phi} \in \mathbb{R}^{K \times 1}$, with $K$ being the total number of discrete experimental observations. In other words, the functional

$$
O F(\mathbf{u})=[\boldsymbol{\Phi}(\mathbf{u})-\mathbf{W}]^{T}[\boldsymbol{\Phi}(\mathbf{u})-\mathbf{W}]
$$

needs to be minimized:

$$
\tilde{\mathbf{u}}=\arg \min _{\mathbf{u}} O F(\mathbf{u}),
$$

with $\tilde{\mathbf{u}}$ being the recovered value of the unknown model parameters. In practice, the actual measurements $\mathbf{W}$ can be expressed as $\mathbf{W}=\boldsymbol{\Phi}\left(\mathbf{u}^{*}\right)+\mathbf{e}$, with e being the uncertainty 'error' vector. A possible difference between the simulated responses $\boldsymbol{\Phi}\left(\mathbf{u}^{*}\right)$ with the actual model parameters $\mathbf{u}^{*}$ and the measurements $\mathbf{W}$ can arise from two reasons, and is denoted by

$$
\mathbf{e}=\mathbf{e}_{n}+\mathbf{e}_{m},
$$

with $\mathbf{e}_{n}$ being the uncertainty due to measurement noise and $\mathbf{e}_{m}$ being the uncertainty due to modeling uncertainties. Due to 


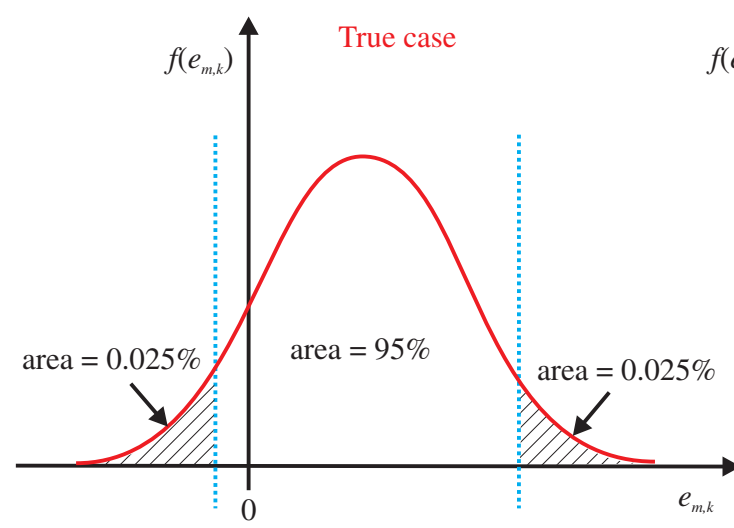

(a)

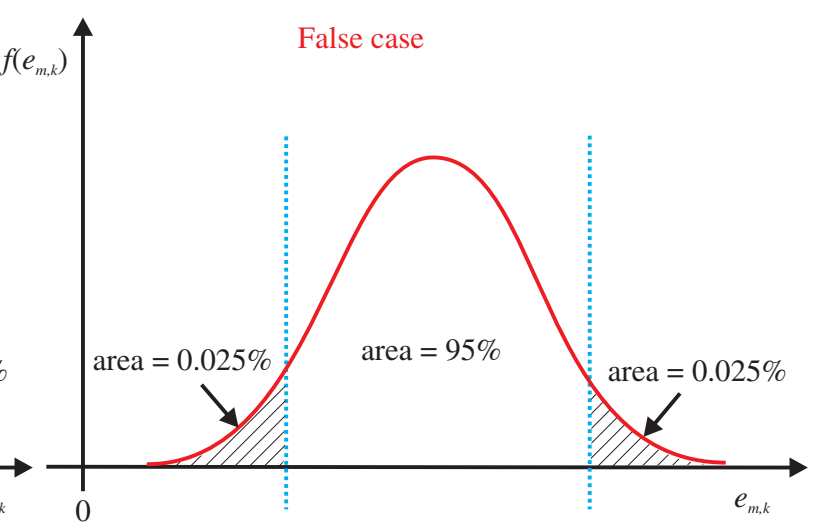

(b)

Figure 1. The normal distribution with the 95\% confidence interval. (a) 'True' case (the probability of observing a zero error is within the 95\% interval), (b) 'False' case (the probability of observing a zero error is outside the $95 \%$ interval).

the random nature of the measurement noise, it is assumed to be normally white distributed with zero mean $\left(\bar{\mu}_{n, k}=0\right)$ and a variance of $\bar{\sigma}_{n, k}^{2}$, i.e. $\left(\mathbf{e}_{n, k} \sim \mathcal{N}\left(0, \bar{\sigma}_{n, k}^{2}\right)\right)$ [6]. Similarly,

$$
\begin{array}{r}
\overline{\boldsymbol{\mu}}_{n}=\left[\mu_{n, 1}, \mu_{n, 2}, \ldots, \mu_{n, K}\right]^{T}=\mathbf{0}, \\
\overline{\boldsymbol{\sigma}}_{n}^{2}=\left[\sigma_{n, 1}^{2}, \sigma_{n, 2}^{2}, \ldots, \sigma_{n, K}^{2}\right]^{T} .
\end{array}
$$

Since the measurement noise is assumed uncorrelated, i.e. $e_{n, i}$ does not depend on $e_{n, j}(i, j=1, \ldots, K, i \neq j)$, the covariance matrix of the measurement noise $\left(\overline{\boldsymbol{\Sigma}}_{n}^{2} \in \mathbb{R}^{K \times K}\right)$ can be written as $\overline{\boldsymbol{\Sigma}}_{n}^{2}=\operatorname{diag}\left(\sigma_{n, 1}^{2}, \sigma_{n, 2}^{2}, \ldots, \sigma_{n, K}^{2}\right)$.

The modeling error is also represented in the normal distribution, see section 2.1. Due to these uncertainties, when minimizing the cost function (5), the values of the recovered parameters $\widetilde{\mathbf{u}}$ and $\mathbf{u}^{*}$ are not necessary equal, i.e. $\widetilde{\mathbf{u}} \neq \mathbf{u}^{*}$. Therefore, we propose the use of the statistical Bayesian approach. In sections 2.2.1 and 2.2.2, a brief summary of the statistical inversion theory is presented. The core of these two sections is mainly based on $[9,16,17]$.

2.2.1. Traditional Bayesian approach. In the Bayesian framework, the identification problem is seen as a statistical inference problem, sometimes referred to as stochastic regularization [18], in which the measurements and the modeled response are assumed to be random [9]. In the traditional Bayesian approach, the modeling error is assumed to be negligible, i.e. $\mathbf{W}=\boldsymbol{\Phi}\left(\mathbf{u}^{*}\right)+\mathbf{e}_{n}$.

In the well-known Bayes' formula, the posterior probability density function of the measurements $\mathbf{W}$ given the unknown model parameters $\mathbf{u}$ ' $P(\mathbf{u} \mid \mathbf{W})$ ' is given by [17]

$$
P(\mathbf{u} \mid \mathbf{W})=\frac{P(\mathbf{u}) P(\mathbf{W} \mid \mathbf{u})}{P(\mathbf{W})},
$$

which can be written in a non-normalized form:

$$
P(\mathbf{u} \mid \mathbf{W}) \propto P(\mathbf{u}) P(\mathbf{W} \mid \mathbf{u}),
$$

with $P(\mathbf{u})$ being the prior probability density function of the unknown model parameters. In our application, no information is given for $P(\mathbf{u})$. So we assume that the unknown model parameters follow the uniform distribution between lower and upper bounds:

$$
P(\mathbf{u})=\frac{1}{\mathbf{u}_{U B}-\mathbf{u}_{L B}}, \quad \mathbf{u} \in\left[\mathbf{u}_{L B}, \mathbf{u}_{U B}\right],
$$

with $\mathbf{u}_{L B}$ and $\mathbf{u}_{U B}$ being the lower and upper bounds of the unknown model parameters, respectively, which can be known from the reasonable physical representation of $\mathbf{u}$.

Assuming that the measurement noise $\mathbf{e}_{n}$ does not depend on the unknown model parameters $\mathbf{u}$, the likelihood density function of the measurements $\mathbf{W}$ given the unknown model parameters $\mathbf{u}$ can be written as [17]

$$
\begin{aligned}
P(\mathbf{W} \mid \mathbf{u})= & \frac{1}{(2 \pi)^{K / 2} \prod_{k=1}^{K} \sigma_{n, k}} \\
& \cdot \exp \left(-\frac{1}{2}\left[\left[\mathbf{W}-\boldsymbol{\Phi}(\mathbf{u})-\overline{\boldsymbol{\mu}}_{n}\right]^{T}\left(\overline{\boldsymbol{\Sigma}}_{n}^{2}\right)^{-1}\right.\right. \\
& {\left.\left.\left[\mathbf{W}-\boldsymbol{\Phi}(\mathbf{u})-\overline{\boldsymbol{\mu}}_{n}\right]\right]\right) . }
\end{aligned}
$$

Therefore, in order to solve this inverse problem, the maximum a posteriori (MAP) estimate is used, in which the MAP of the unknown model parameters $\mathbf{u}$ is given by

$$
\mathbf{u}_{\mathrm{MAP}}=\arg \max _{\mathbf{u}} P(\mathbf{u} \mid \mathbf{W}) \text {. }
$$

Substituting (10) and (12) into (13), and $\left(\overline{\boldsymbol{\mu}}_{n}=0\right)$,

$$
\begin{aligned}
\mathbf{u}_{\text {MAP,Trad }}= & \arg \max _{\mathbf{u}} P(\mathbf{W} \mid \mathbf{u}) \\
= & \arg \max _{\mathbf{u}}\left\{\operatorname { e x p } \left(-\frac{1}{2}\left[[\mathbf{W}-\boldsymbol{\Phi}(\mathbf{u})]^{T}\left(\overline{\mathbf{\Sigma}}_{n}^{2}\right)^{-1}\right.\right.\right. \\
& \times[\mathbf{W}-\boldsymbol{\Phi}(\mathbf{u})]])\} \\
= & \arg \min _{\mathbf{u}}\left\{[\mathbf{W}-\boldsymbol{\Phi}(\mathbf{u})]^{T}\left(\overline{\boldsymbol{\Sigma}}_{n}^{2}\right)^{-1}[\mathbf{W}-\boldsymbol{\Phi}(\mathbf{u})]\right\} \\
= & \arg \min _{\mathbf{u}}\left\|\mathbf{L}_{n}(\mathbf{W}-\boldsymbol{\Phi}(\mathbf{u}))\right\|^{2},
\end{aligned}
$$

with $\mathbf{L}_{n}$ being the Cholesky factor of the covariance of the measurement noise, i.e. $\left(\overline{\boldsymbol{\Sigma}}_{n}^{2}\right)^{-1}=\mathbf{L}_{n}^{T} \mathbf{L}_{n}$. The solution of (14) is the recovered model parameter $\left(\mathbf{u}_{\text {MAP,Trad }} \equiv \widetilde{\mathbf{u}}\right)$ using the inverse problem in the traditional Bayesian framework.

2.2.2. Bayesian approximation error approach. In the traditional Bayesian approach, the modeling error $\mathbf{e}_{m}$ was assumed to be negligible. However, in the Bayesian approximation error approach, $\mathbf{e}_{m}$ is taken into account. As 
discussed earlier in section 2.2, the modeling error exists only when the coarse model is incorporated in the inverse problem, so the forward model can be rewritten as $\mathbf{W}=$ $\boldsymbol{\Phi}_{c}\left(\mathbf{u}^{*}\right)+\mathbf{e}_{n}+\mathbf{e}_{m}$. Due to the Gaussian distribution of both the measurement noise and modeling error, the overall error $\mathbf{e}$ is therefore also Gaussian distributed. Similarly, (14) can be reformulated as follows:

$$
\begin{aligned}
\mathbf{u}_{\text {MAP,Compensated }} & =\arg \max _{\mathbf{u}} P(\mathbf{W} \mid \mathbf{u}) \\
& =\arg \min _{\mathbf{u}}\left\|\mathbf{L}_{n+m}\left(\mathbf{W}-\boldsymbol{\Phi}_{c}(\mathbf{u})-\overline{\boldsymbol{\mu}}_{m}\right)\right\|^{2},
\end{aligned}
$$

with $\mathbf{L}_{n+m}$ being the Cholesky factor of the covariance of the overall error, i.e. $\left(\overline{\boldsymbol{\Sigma}}_{n}^{2}+\overline{\boldsymbol{\Sigma}}_{m}^{2}\right)^{-1}=\mathbf{L}_{n+m}^{T} \mathbf{L}_{n+m}$. The solution of (15) is the recovered model parameter $\left(\mathbf{u}_{\text {MAP,Compensated }} \equiv \widetilde{\mathbf{u}}\right)$ using the inverse problem in the Bayesian approximation error approach, in which the modeling error is compensated. It is worth mentioning that under the assumption of uncorrelatedness, the covariance matrices are diagonal, i.e. the Cholesky factorization is reduced to taking the square root.

Note that, if it is possible to solve traditionally the inverse problem, the Bayesian approximation error (15) can then be solved, provided enough $Z$ samples for calculating the $\overline{\boldsymbol{\mu}}_{m}$ and $\mathbf{L}_{n+m}$ using (2). In the case of high-dimensional inverse problems, i.e. a high number of parameters need to be recovered, a large number of evaluations in the forward models (1) are needed, since the larger the number of parameters, the larger the $Z$. A possible limitation of the methodology is thus that the methodology becomes computationally prohibitive to calculate in the case of high-dimensional inverse problems. The high-dimensionality challenge is notorious and a possibility of improving the Bayesian approximation error approach when solving highdimensional inverse problems is to use one of the following strategies: the reduction of design space, decomposition of problems into sub-problems, screening of the significant variables, etc [23]. Other possibilities are the use of distributed computing and adaptive sampling based on $e_{m, k}$ outputs for the reduction of the number of evaluations in the forward models.

\section{EMD modeling}

There are several techniques to model an EMD. Basically, these techniques can be subdivided into two main categories: numerical (e.g. finite element, finite difference, boundary elements, etc) and analytical techniques. Apart from numerical techniques, the finite-element method is commonly used for modeling EMDs. In this section, we propose three different models of an EMD, sections 3.2-3.4. These models are chosen with different accuracy levels. In all these models, the magnetic material is modeled as presented in section 3.1.

\subsection{Magnetic material modeling}

For all the EMD models, to be mentioned in sections 3.23.4 , the normal magnetizing $B-H$ curve of the core material is characterized by [6]

$$
\frac{H}{H_{0}}=\left(\frac{B}{B_{0}}\right)+\left(\frac{B}{B_{0}}\right)^{v},
$$

with the parameters $\mathbf{u}=\left[H_{0}(\mathrm{~A} / \mathrm{m}), B_{0}(\mathrm{~T}), v\right]$. The values of these parameters are unknown and need to be identified using an inverse approach.

\subsection{D-FE model}

As a very fine model, the numerical model of an EMD is constructed using the 3D-FE model which solves the nonlinear static Maxwell's equation:

$$
\nabla \times\left(\frac{1}{\mu_{0} \mu_{r}(\mathbf{A})} \nabla \times \mathbf{A}\right)=\mathbf{J}
$$

for the magnetic vector potential $\mathbf{A}$ with the nonlinear relative magnetic permeability $\mu_{r}=B /\left(\mu_{0} H\right)$, defined by (16), with $\mu_{0}$ being the magnetic permeability of air, and the current density $\mathbf{J}$, which is related to the enforced current in the excitation windings. In the 3D-FE model, $\mathbf{J}$ and hence magnetic field and induction have three components, i.e. $J_{x}, J_{y}$ and $J_{z}\left(\mathbf{J}=J_{x} \mathbf{i}_{x}+J_{y} \mathbf{i}_{y}+J_{z} \mathbf{i}_{z}\right)$. The EMD can be discretized into several tetrahedrons. The degree of the model fineness, and consequently the complexity, increases with the increase of the density of the mesh discretization.

\subsection{D-FE model}

Due to the fact that the construction of the 3D-FE model is usually a hard task, and the computational time is too expensive, the simplified 2D-FE model is commonly used for modeling an EMD. The 2D-FE model solves the nonlinear static Maxwell's equation (17). However, in this case, the current density $\mathbf{J}$ is perpendicularly oriented in the plane of the magnetic circuit $\left(\mathbf{J}=J_{z} \mathbf{i}_{z}\right.$ being the current density in the $z$-direction). Consequently, the magnetic induction and field are also oriented in this plane. The vector potential has thus a component perpendicular to the plane of the magnetic circuit: $\mathbf{A}=A_{z} \mathbf{i}_{z}$. The Maxwell equation (17) can in this way be reduced to

$$
\nabla \times\left(\frac{1}{\mu_{0} \mu_{r}\left(A_{z}\right)} \nabla \times A_{z}\right)=-J_{z} .
$$

The EMD is discretized here into several triangles. As mentioned in the 3D-FE, the degree of the model fineness, and consequently the complexity, increases with increasing the density of the mesh discretization. When using 2D-FE models, the EMD is assumed to be infinitely long in the $z$-direction and thus a simplification of the 3D-FE models. Hence, the 3D stray magnetic fluxes are not modeled properly in 2D-FE models. Therefore, the 3D-FE models are more accurate than the 2DFE models, but are computationally more demanding.

\subsection{An analytical model}

In order to save computational time, analytical models can be used for modeling an EMD. These analytical models are much faster than numerical models; however, they are less accurate, especially for complex geometries. In this paper, we propose the use of an analytical model of the EI core inductor, which is based on the magnetic reluctance network theory [19]. In these analytical models, the EMD is approximated by a magnetic network of reluctances and a magneto-motive force 
as a source. The magnetic network is constructed in such a way that every geometrical part of the magnetic circuit of the EMD, in which we assume a uniform field pattern, is substituted by a certain reluctance.

\section{Results and discussion}

In order to validate the presented methodology, we study two different geometries of EMDs. In section 4.1, the investigation is performed in a purely numerical way, where no real measurements are used, to identify the magnetic material properties of the SRM core material. Then, in section 4.2, we solve an inverse problem starting from real measurements in order to recover the magnetic material parameter values of an EI core inductor.

The two EMDs are chosen in such a way to have completely different objective function formulations of the inverse problem. The inverse problem of the SRM is based on the mechanical output, whereas the inverse problem of the EI core inductor is based on magnetic measurements. From our experience [7], we discovered that the noise in the mechanical measurements is relatively high and that it may be the dominant error source. In practice, it is difficult to precisely quantify the measurement noise. Since we aim at reducing the error originating from the simplified coarse model in the inverse problem, we validate the proposed methodology in a numerical way (numerical data as input to the inverse problem) using the SRM inverse problem. Moreover, the methodology is validated in an experimental way (real experimental data as input to the inverse problem) using the EI core inductor inverse problem.

In the two case studies, we use the well-known leastsquares nonlinear algorithm, Levenberg-Marquardt method with line search [20]. Note that the used optimization scheme depends on the considered problem. If multiple local minima exist, it is possible to use multiple starting values within the deterministic optimization scheme (e.g. least-squares nonlinear, gradient-based methods) or employ a time-demanding stochastic optimization scheme (e.g. genetic algorithm) so as to recover the global optimum.

The inverse problem in this paper is well-posed since the number of parameters to be recovered $(p=3)$ is much less than the number of measurements $(K=19$ in the SRM application, $K=14$ in the EI core application). In the case of ill-posed inverse problems, (14) or (15) becomes more difficult to solve. In that case, it is necessary to use regularization techniques such as the Tikhonov regularization technique within the cost functional (14) or (15).

\subsection{Case study (1): a SRM}

For this case study, we build the following two computer models: a fine model based on 2D-FE with fine mesh discretizations and a coarse model based on a magnetic reluctance network, see [15].

4.1.1. Studied geometry. Figure 2 shows the schematic diagram of the 6/4 SRM. The geometry is characterized by the following geometrical parameters: $t_{s p}, t_{r p}, D_{r i}, D_{r e}$,

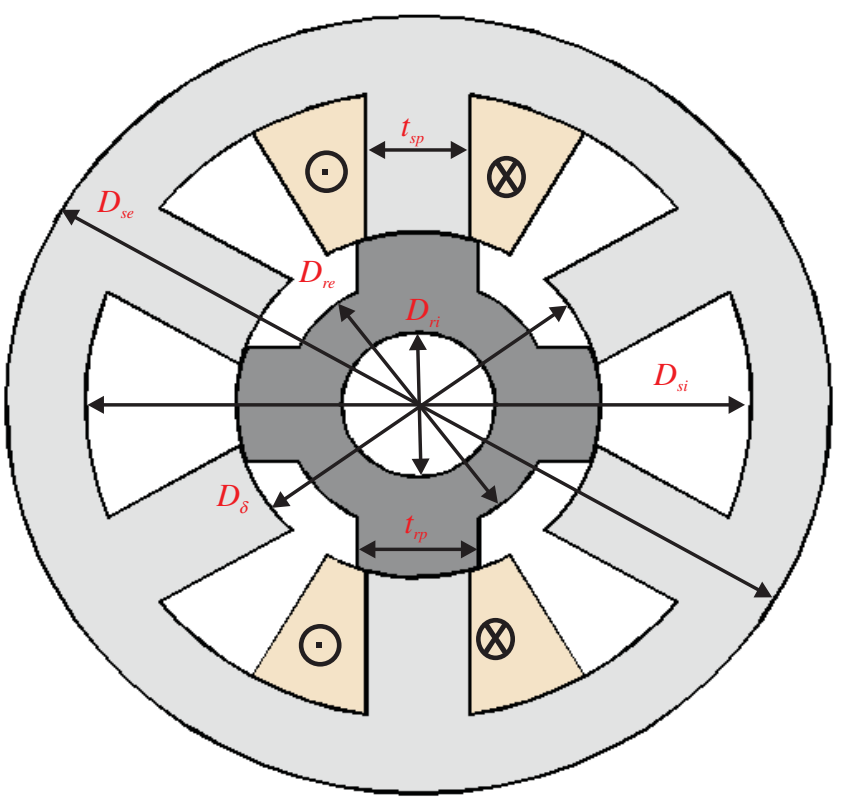

Figure 2. Schematic diagram of the studied 6/4 SRM. $\delta$ is the air gap at the alignment condition. The length of the motor core is $63.5 \mathrm{~cm}$.

$D_{\delta}, D_{s i}, D_{s e}, \delta$, where $t_{s p}$ and $t_{r p}$ are the stator and the rotor pole width, $D_{r i}$ and $D_{r e}$ are the internal and external diameter of the rotor yoke, respectively, $D_{\delta}$ is the inner stator pole diameter, $D_{s i}$ and $D_{s e}$ are respectively the internal and external diameters of the stator yoke and $\delta$ is the air gap thickness. The values of the geometrical parameters are $\mathbf{d}=\left[t_{s p}, t_{r p}, D_{r i}, D_{r e}, D_{\delta}, D_{s i}, D_{s e}, \delta\right]=$ $[17,20,25,44,60,109.2,135,0.25] \mathrm{mm}$.

4.1.2. Inverse problem formulation for the SRM. In order to identify the magnetic material parameter values $\mathbf{u}$ of the SRM, an inverse problem is solved. In this inverse problem, an objective function is formulated, which minimizes iteratively the quadratic difference between the measured and simulated response. Here, in this case study, we consider the static torque profile at a fixed excitation current $I_{0}=8 \mathrm{~A}$, and for $K=19$ mechanical rotor angles $\xi=\left[0^{\circ}, 2.5^{\circ}, \ldots, 45^{\circ}\right]$. The static torque value corresponding to the $k$ th rotor angle $\xi_{k}$ can be computed using [7]

$$
T_{k}(\mathbf{u})=\left.\frac{\partial}{\partial \xi} \Upsilon_{\mathrm{co}}(\xi, \mathbf{u})\right|_{\left(\xi=\xi_{k}, I_{0}=8 A\right)},
$$

where $\Upsilon_{\text {co }}$ is the co-energy for a certain fixed excitation current $I_{0}$.

As no real measurements are performed, the values of the material parameters are assumed to be known $\left(\mathbf{u}^{*}=\right.$ $[231.6,1.34,15.18])$. These parameter values are used as an input of the fine model to generate the 'numerical measurement' of the static torque profile of the SRM. The 'numerical measurement' is corrupted by Gaussian noise with zero mean and a standard deviation of $\sigma_{n}$, which is given by $\sigma_{n}=\left(n l T_{\text {rms }}\right) \times \mathcal{N}[0,1]$, where $n l$ is the noise level in the measurement. $T_{\mathrm{rms}}$ is the root mean square of the 'numerically measured' static torque profile: $T_{\mathrm{rms}}=\sqrt{\frac{1}{K} \sum_{k=1}^{K} T_{k}^{2}} \cdot \mathcal{N}[0,1]$ is a normally distributed random number with zero mean and 


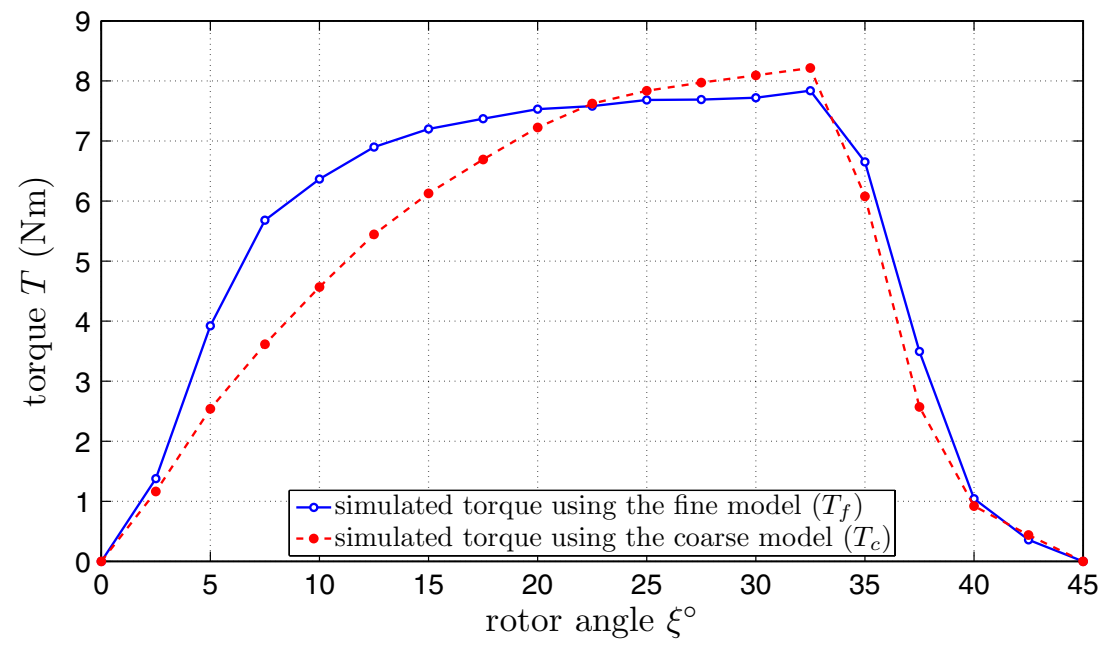

Figure 3. The simulated torque profile using the fine and coarse model, $T_{f}$ and $T_{c}$, respectively. Both torque profiles are obtained using $\left(\mathbf{u}^{*}=[231.6,1.34,15.18]\right)$.

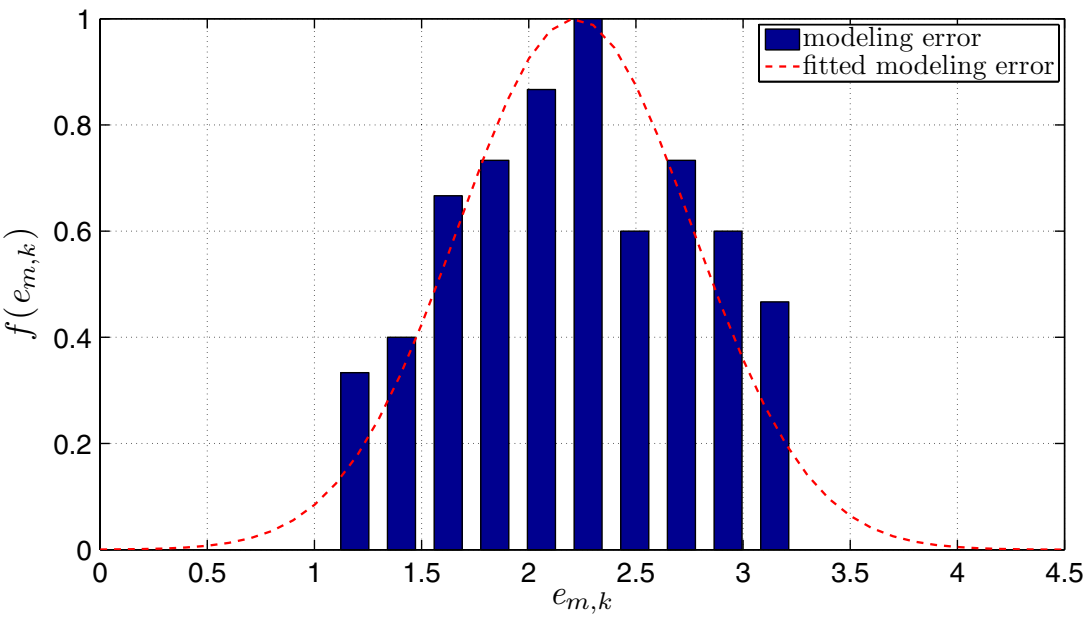

Figure 4. The stochastic representation of the misfit between the 2D-FE and the analytical models for the output static torque profile of the studied SRM.

a standard deviation of 1 . Therefore, equations (14) and (15) can be rewritten as

$\mathbf{u}_{\text {MAP,Trad,SRM }}=\arg \min _{\mathbf{u}}\left\|\mathbf{L}_{n}\left(\mathbf{T}_{f}\left(\mathbf{u}^{*}\right)-\mathbf{T}_{c}(\mathbf{u})\right)\right\|^{2}$

$$
\begin{array}{r}
\mathbf{u}_{\text {MAP,Compensated,SRM }}=\arg \min _{\mathbf{u}} \| \mathbf{L}_{n+m}\left(\mathbf{T}_{f}\left(\mathbf{u}^{*}\right)\right. \\
\left.-\mathbf{T}_{c}(\mathbf{u})-\overline{\boldsymbol{\mu}}_{m}\right) \|^{2},
\end{array}
$$

with $\mathbf{L}_{n}$ and $\mathbf{L}_{n+m}$ being the Cholesky factors of the covariance of the measurement noise and the overall error, respectively, i.e. $\left(\overline{\boldsymbol{\Sigma}}_{n}^{2}\right)^{-1}=\mathbf{L}_{n}^{T} \mathbf{L}_{n},\left(\overline{\boldsymbol{\Sigma}}_{n}^{2}+\overline{\mathbf{\Sigma}}_{m}^{2}\right)^{-1}=\mathbf{L}_{n+m}^{T} \mathbf{L}_{n+m}$.

Based on the recovered $B-H$ curve at each inverse problem compared to the original $B-H$ curve, one may calculate the recovery error (RE). Many formulas can be used; however, we calculate RE based on the ratio between the area under the recovered and the original $B-H$ curves, respectively [7]:

$$
\mathrm{RE}=\left(\frac{\int_{0}^{H_{\max }} B_{\text {recovered }} \cdot \mathrm{d} H}{\int_{0}^{H_{\max }} B_{\text {original }} \cdot \mathrm{d} H}-1\right)^{2} \times 100 \%,
$$

where $H_{\max }$ is the maximum magnetic field. Here it is assumed $H_{\text {max }}=2000 \mathrm{Am}^{-1}$.
4.1.3. Stochastic representation of the modeling error in a $S R M$. In order to represent the modeling error between the fine and the coarse model stochastically, the procedure presented in section 2.1 is used. Several sampling techniques can be used for generating the $Z$ hypothetical values of $\mathbf{u}$. We use here the Latin hypercube sampling (LHS) [21], which is a widely used statistical method for generating a certain set of samples $\left(\left\{\mathbf{u}_{1}, \ldots, \mathbf{u}_{Z}\right\}\right)$ for a given domain. $Z$ needs to be taken high enough so that an as accurate as possible Gaussian fit of the modeling error can be performed.

$Z$ forward fine and coarse model computations are solved, for the input parameters $\mathbf{u}_{i}, i=1, \ldots, Z$, and their static torque values $T_{f, k}\left(\mathbf{u}_{i}\right), T_{c, k}\left(\mathbf{u}_{i}\right)(k=1, \ldots, K)$ are compared. Figure 3 shows the simulated torque profile using the fine and coarse model, $T_{f}$ and $T_{c}$, respectively. Here, both static torque profiles are obtained using the $\mathbf{u}^{*}$. Figure 4 depicts the stochastic behavior of the modeling error of the coarse model compared to the fine model using (1)-(3), at a certain mechanical rotor angle. It is clear from figure 4 that the stochastic variation of the modeling error can be 'approximated' by a Gaussian distribution. 


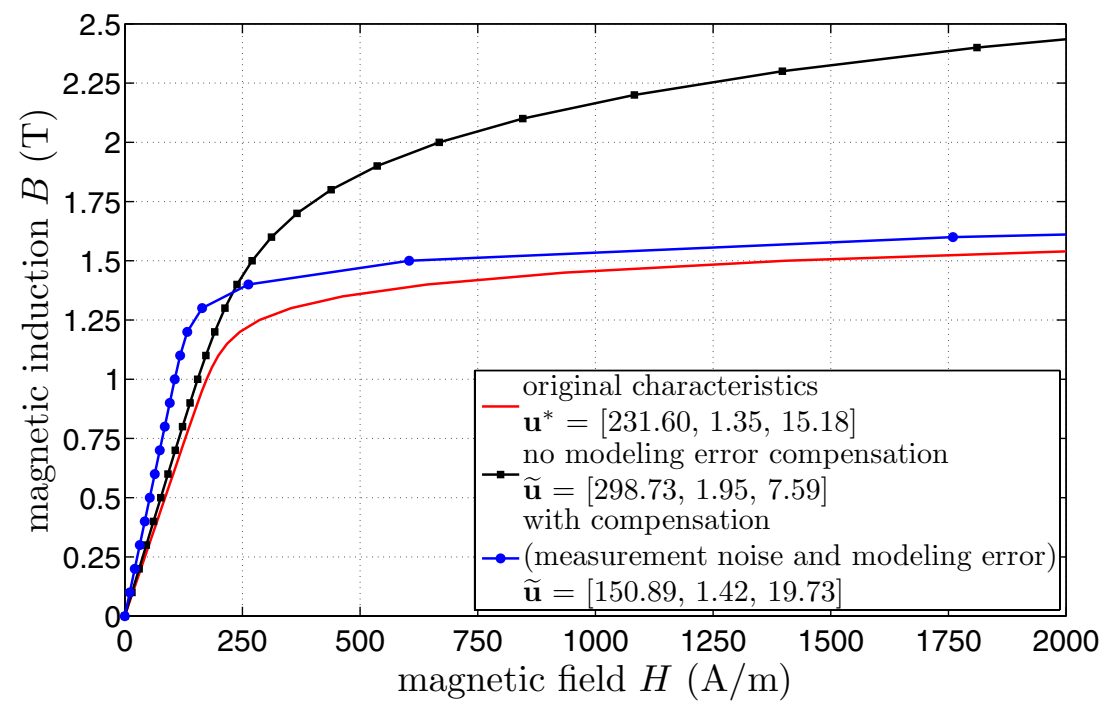

Figure 5. The recovered $B-H$ curve using the two inverse problems based on the traditional Bayesian approach 'no modeling error compensation' and the Bayesian approximation error approach 'with modeling error compensation' compared to the original characteristics.

4.1.4. Theoretical results. In this section, two cases of the modeling error are discussed. In the first case, the modeling error is assumed to be uncorrelated, while in the second case, we consider the correlation in the modeling error.

(i) Uncorrelated modeling error: under the assumption of uncorrelated modeling error and starting from noisy 'numerical' measurements with noise level $n l=10 \%$, the inverse problem is solved using two approaches. The first methodology is based on the traditional Bayesian approach (20), in which the modeling error is not compensated. The second inverse approach is based on the Bayesian approximation error approach (21) with the modeling error compensation. Figure 5 illustrates the solution of the two inverse methodologies compared to the original characteristics. It is clear from figure 5 that the inverse problem based on (21) gives better results compared to the one based on (20). The recovery error values (RE) for the traditional and the Bayesian approximation error approaches are $20.5234 \%$ and $0.5491 \%$, respectively. Also, it is worth mentioning that the computational time required for both approaches (20) and (21) is approximately the same since the modeling error term is computed in advance. The results presented in this section validate 'theoretically' the proposed approach.

(ii) Correlated modeling error: the modeling error is often highly correlated. For the simplicity of the analysis, the 'numerical' measurements are assumed noise free. Therefore, equations (14) and (15) can be rewritten as $\left(\mathbf{L}_{n}=\mathbf{I}_{K}\right.$ with $\mathbf{I}_{K}$ being the $K$ th-dimensional identity matrix)

$\mathbf{u}_{\text {MAP,Trad,SRM }}=\arg \min _{\mathbf{u}}\left\|\mathbf{T}_{f}\left(\mathbf{u}^{*}\right)-\mathbf{T}_{c}(\mathbf{u})\right\|^{2}$

$$
\begin{array}{r}
\mathbf{u}_{\text {MAP,Compensated,SRM }=} \arg \min _{\mathbf{u}} \| \mathbf{L}_{m}\left(\mathbf{T}_{f}\left(\mathbf{u}^{*}\right)\right. \\
\left.-\mathbf{T}_{c}(\mathbf{u})-\overline{\boldsymbol{\mu}}_{m}\right) \|^{2},
\end{array}
$$

with $\mathbf{L}_{m}$ being the Cholesky factor of the covariance of the modeling error, i.e. $\left(\overline{\boldsymbol{\Sigma}}_{m}^{2}\right)^{-1}=\mathbf{L}_{m}^{T} \mathbf{L}_{m}$.

We solve three types of inverse problems. The first one is based on (23) where the modeling error is not compensated. The second and third inverse problems are solved with the compensation of the modeling error (see equation (24)) and is assumed uncorrelated and correlated, respectively. When assuming an uncorrelated modeling error, the covariance matrix is diagonal and the Cholesky factorization is reduced to taking the square root. In the third inverse problem, we use the full correlation matrix of the modeling error. We calculate the covariance matrix of the modeling error as (see also equation (2) for the calculation of the diagonal elements of the covariance matrix)

$$
\begin{aligned}
\operatorname{Cov}\left(e_{m, k}, e_{m, l}\right)=\frac{1}{Z} & \sum_{z=1}^{Z}\left(e_{m, k}\left(\widehat{\mathbf{u}}_{z}\right)-\mu_{m, k}\right) \\
& \times\left(e_{m, l}\left(\widehat{\mathbf{u}}_{z}\right)-\mu_{m, l}\right) .
\end{aligned}
$$

Figure 6 shows the reconstructed $B-H$ characteristics for the three different inverse problems. We observe that when assuming correlated modeling errors, the characteristic is more accurately recovered compared to when assuming uncorrelated modeling errors. However, the increase in accuracy between the second and third inverse solution is much smaller compared to the increase in accuracy of the first inverse solution versus the second and third inverse solution. The recovery error values (RE) for the three inverse problems are $8.5201 \%, 0.1475 \%$ and $0.0011 \%$, respectively.

\subsection{Case study (2): an EI core inductor}

In this specific case, we build three computer models: a very fine model based on 3D-FE with fine mesh discretizations (model a), a moderate fine model based on 3D-FE with coarse 


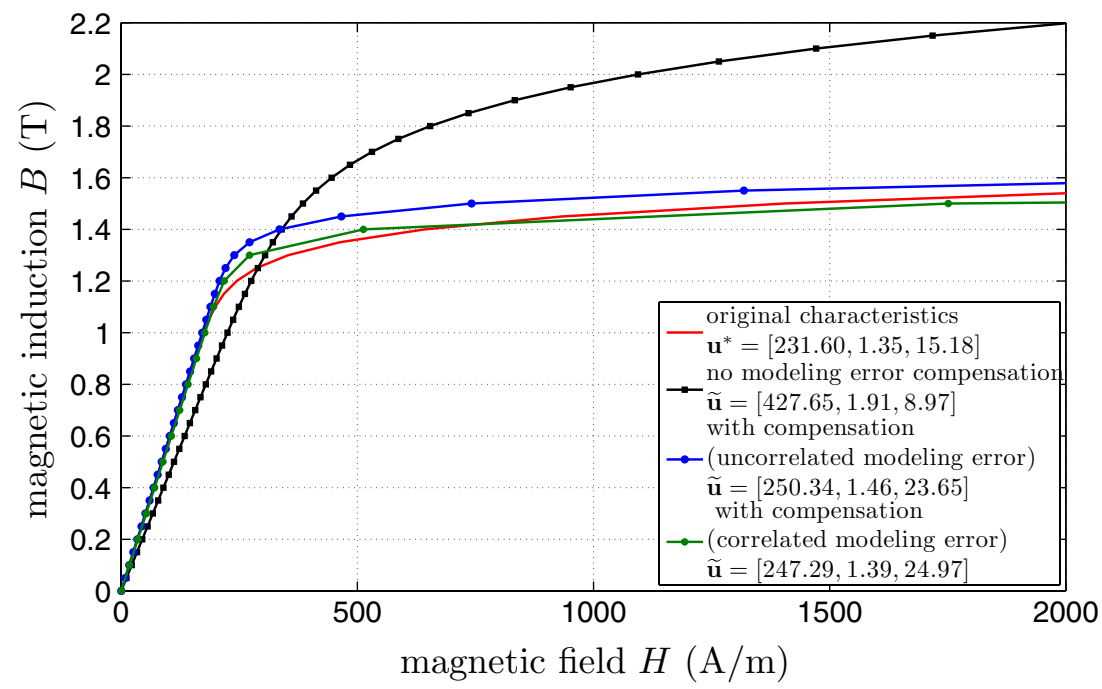

Figure 6. The recovered $B-H$ curve using (23) with no modeling error compensation and using (24) with modeling error compensation (assumed uncorrelated and correlated modeling errors).

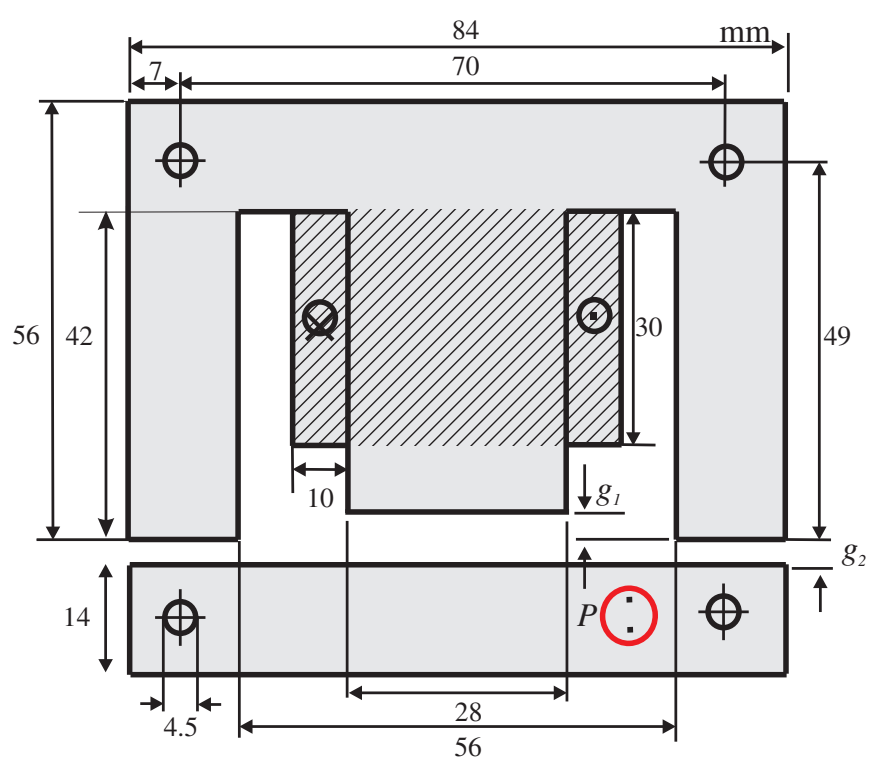

Figure 7. Schematic diagram of the studied EI electromagnetic core inductor. At position $P$, the local magnetic induction measurements are carried out.

mesh discretizations (model b) and a coarse model based on a magnetic reluctance network (model $\mathrm{c}$ ) in a similar way as presented in [22]. Here, we consider 'model a' as the fine model; however, the other two models, i.e. 'model b' and 'model c', are considered as 'relatively' coarse models.

4.2.1. Studied geometry. Figure 7 shows the profile of the studied geometry. It is an EI core with one 'middle' air gap in the middle limb $\left(g_{1}\right)$ and two 'outer' air gaps $\left(g_{2}\right)$ between Eand I-yokes. The excitation coil is wound around the middle limb of the E-core with 356 excitation winding turns. The dimensions of the EI core inductor are precisely known, as shown in figure 7. The value of $g_{1}$ is $0.85 \mathrm{~mm}$. However, the value of $g_{2}$ is kept zero in order to eliminate the modeling uncertainty caused by the uncertain value of $g_{2}$, see [6]. The two yokes are fixed together by a mechanical clamp to prevent the movement of the I-yoke.

\subsubsection{Inverse problem formulation for the EI core inductor.} In order to identify the magnetic material parameter values $\mathbf{u}$ of the EI core inductor, an inverse problem is solved. In this inverse problem, an objective function is formulated, which minimizes iteratively the quadratic difference between the measured and simulated response. Here, in this case study, we consider the local magnetic induction at position $P$ (see figure 7) for $K=14$ amplitudes of the sinusoidal excitation current.

The local magnetic induction is measured using the needle probe method. Indeed, the voltage between the needles is proportional to the rate of change of the local magnetic induction [5]. We performed these real measurements at position $P$ as shown in figure 7 . The quasi-static magnetic measurements are performed at $1 \mathrm{~Hz}$ for a sinusoidal current excitation, in order to have a negligible skin effect due to eddy currents in the magnetic cores, see [5]. We choose the position on the I-yoke, i.e. $P$ in figure 7 , because at this position the best inverse problem resolution is observed, see the results stated in [6].

The measurements are carried out five times and the average measurement values are used in the inverse problem. In fact, very small variations are noted among the five local measurements. Therefore, we consider the measurements as being noise-free measurements, i.e. $\mathbf{e}_{n}=0$. This assumption is reasonable because the local magnetic induction measurements contain only a very limited amount of noise. In addition, this small measurement noise is difficult to quantify precisely in practice. Hence, (14) and (15) are reformulated as follows:

$$
\begin{aligned}
& \mathbf{u}_{\text {MAP,Trad,EI }}=\arg \min _{\mathbf{u}}\left\|\mathbf{B}_{\mathrm{m}}-\mathbf{B}_{\mathrm{S}}(\mathbf{u})\right\|^{2} \\
& \mathbf{u}_{\text {MAP,Compensated,EI }}=\arg \min _{\mathbf{u}} \| \mathbf{L}_{m}\left(\mathbf{B}_{\mathrm{m}}\right. \\
& \left.-\mathbf{B}_{\mathrm{S}}(\mathbf{u})-\overline{\boldsymbol{\mu}}_{m}\right) \|^{2}
\end{aligned}
$$




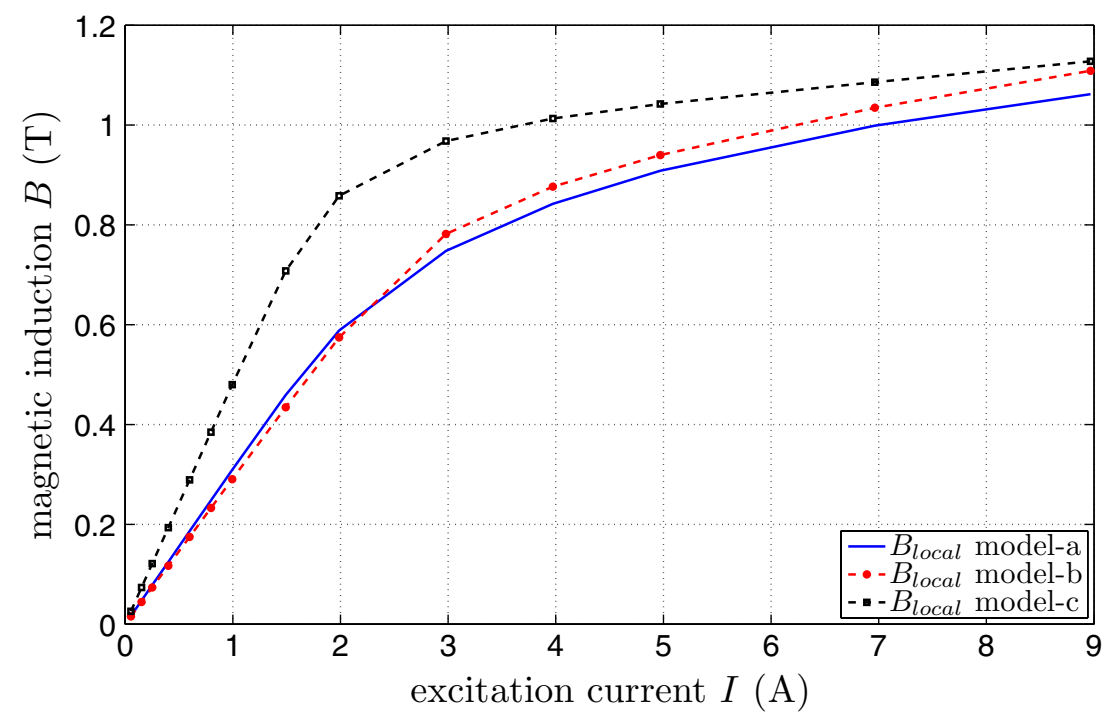

Figure 8. The simulated local magnetic induction using the three computer models. All profiles are obtained using $(\widehat{\mathbf{u}}=[1291.2,1.197,10.28])$.

where $\mathbf{B}_{\mathrm{m}}$ is the measured local magnetic induction at position $P . \mathbf{B}_{\mathrm{S}}$ is the corresponding simulated local magnetic induction at position $P$ in figure 7. Equation (26) is solved for the three computer models; however, (27) is solved only for the two 'relative' coarse computer models, i.e. 'model b' and 'model c', because no modeling error is considered in 'model a'.

\subsubsection{Stochastic representation of the modeling error in} an EI core inductor. In order to represent the modeling error between the fine and the coarse model stochastically, the procedure presented earlier in section 2.1 is used. $Z$ hypothetical values of $\mathbf{u}$ are generated using the LHS technique.

$Z$ forward fine and coarse model computations are solved, and their responses 'local magnetic induction measurements' (i.e. $\mathbf{B}_{s}$ ) are compared. Figure 8 shows the simulated local magnetic induction using the three models a, b and c. All these simulation results are obtained using a certain hypothetical value of $\mathbf{u}$, i.e. $\widehat{\mathbf{u}}=[1291.2,1.197,10.28]$.

Initially, it is clear from figure 8 that model $\mathrm{b}$ gives a better 'forward problem' response than model c. In order to quantify the degree of the coarseness of each model, the 95\% confidence interval presented in section 2.1.1 is used. Table 1 shows the mean and the standard deviation values and the corresponding 'True' (T) and 'False' (F) cases for both models. The percentage degrees of the model coarseness $\eta$ for models b and c are $14.29 \%$ and $64.29 \%$, respectively. Hence, we expect that model $b$ will give much better results than model c. Moreover, we expect that the Bayesian approach will not be able to reduce the modeling error, originating from the simplification of model c, to an 'acceptable' recovery error, see the following section.

4.2.4. Experimental validation. The recovered magnetic material characteristics using the inverse problems are compared with the original normal magnetizing $B-H$ curve of the material, which is measured using the IEEE standard
Table 1. The mean and the standard deviation values $\left(\times 10^{-3}\right)$, and the corresponding 'True' $(\mathrm{T})$ and 'False' $(\mathrm{F})$ cases of the modeling error for models $\mathrm{b}$ and $\mathrm{c}$.

\begin{tabular}{rrrllll}
\hline$k$ & \multicolumn{3}{c}{ Model b } & \multicolumn{3}{c}{ Model c } \\
& \multicolumn{1}{c}{$\mu_{k}$} & \multicolumn{1}{c}{$\sigma_{k}$} & (T or F) & $\mu_{k}$ & \multicolumn{1}{l}{$\sigma_{k}$} & $(\mathrm{~T}$ or F) \\
\hline 1 & 0.8 & 0.2 & $\mathrm{~T}$ & -19.3 & 24.2 & $\mathrm{~T}$ \\
2 & 2.2 & 0.6 & $\mathrm{~T}$ & -22.6 & 2.8 & $\mathrm{~F}$ \\
3 & 3.6 & 1.0 & $\mathrm{~T}$ & -37.3 & 4.6 & $\mathrm{~F}$ \\
4 & 5.8 & 1.7 & $\mathrm{~F}$ & -59.3 & 7.3 & $\mathrm{~F}$ \\
5 & 8.7 & 2.5 & $\mathrm{~F}$ & -88.8 & 10.9 & $\mathrm{~F}$ \\
6 & 11.5 & 3.3 & $\mathrm{~F}$ & -118.2 & 14.5 & $\mathrm{~F}$ \\
7 & 14.1 & 4.2 & $\mathrm{~T}$ & -147.7 & 18.0 & $\mathrm{~F}$ \\
8 & 19.0 & 7.1 & $\mathrm{~F}$ & -213.1 & 29.3 & $\mathrm{~F}$ \\
9 & 19.2 & 11.0 & $\mathrm{~T}$ & -246.7 & 55.7 & $\mathrm{~F}$ \\
10 & 9.0 & 12.0 & $\mathrm{~T}$ & -232.9 & 78.4 & $\mathrm{~F}$ \\
11 & -0.7 & 24.4 & $\mathrm{~T}$ & -199.8 & 81.1 & $\mathrm{~F}$ \\
12 & -7.4 & 32.7 & $\mathrm{~T}$ & 171.9 & 76.5 & $\mathrm{~F}$ \\
13 & -24.6 & 61.6 & $\mathrm{~T}$ & -145.1 & 72.1 & $\mathrm{~F}$ \\
14 & -48.0 & 102.4 & $\mathrm{~T}$ & -145.1 & 110.8 & $\mathrm{~T}$ \\
\hline
\end{tabular}

393-1991, see [5]. The original $B-H$ curve is fitted by equation (16), which results in the actual material parameter values $\mathbf{u}^{*}=$ [292 $\left.\left(\mathrm{A} \mathrm{m}^{-1}\right), 1.35(\mathrm{~T}), 11.99\right]$.

Different inverse problems are solved, with the assumption of $\mathbf{e}_{n}=0$, for each computer model. Then, the identified magnetic characteristics (single-valued $B-H$ curve) are compared. For model a, only one inverse problem is solved based on the traditional Bayesian approach (26). However, for models b and c, four inverse problems are solved, two for each computer model. The first inverse problem is based on the traditional Bayesian approach (26), in which the modeling error is not compensated, while the other inverse problem is based on the Bayesian approximation error approach (27), in which the modeling error is compensated.

Figure 9 illustrates the solution of the five inverse problems compared to the original characteristics. It is clear from figure 9 that the inverse problem based on (27) gives better results compared to the one based on (26) for both 


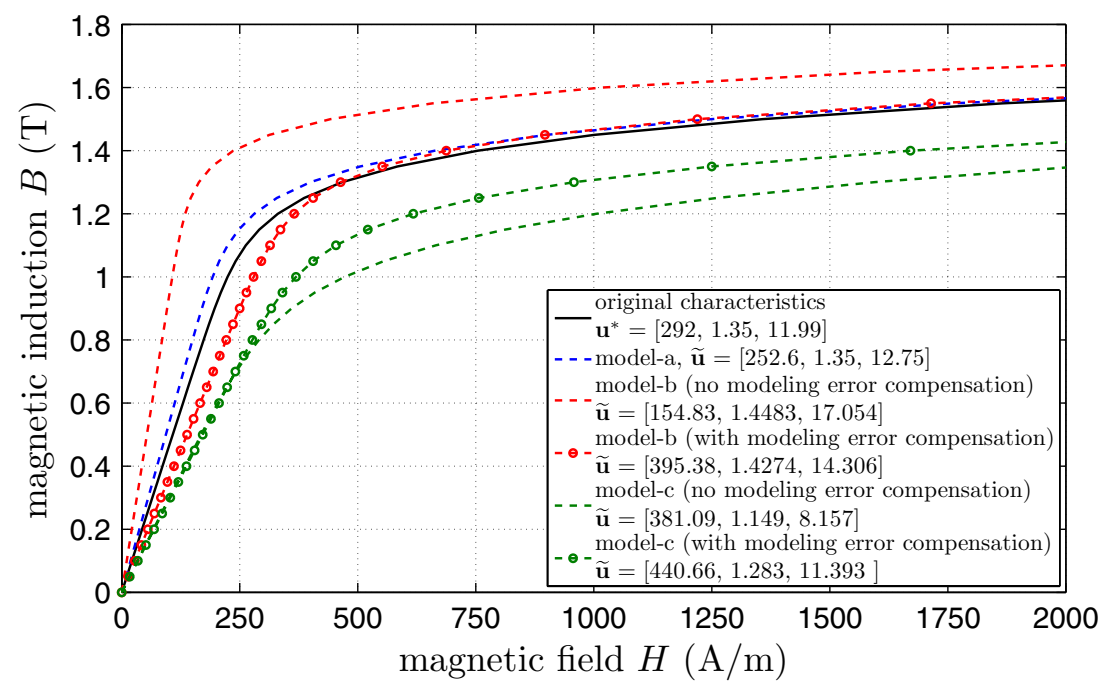

Figure 9. The recovered $B-H$ curve using the two inverse problems based on the traditional Bayesian approach 'no modeling error compensation' and the Bayesian approximation error approach 'with modeling error compensation' for models b and c, and the recovered characteristics for model a, compared to the original characteristics.

Table 2. The values of the recovery error for the three models.

\begin{tabular}{llllll}
\hline Model & Model a & $\begin{array}{l}\text { Model b } \\
\text { (no } \\
\text { compensation) }\end{array}$ & $\begin{array}{l}\text { Model b } \\
\text { (with } \\
\text { compensation) }\end{array}$ & $\begin{array}{l}\text { Model c } \\
\text { (no } \\
\text { compensation) }\end{array}$ & $\begin{array}{l}\text { Model c } \\
\text { (with } \\
\text { compensation) }\end{array}$ \\
\hline RE \% & 0.0266 & 2.0399 & 0.0161 & 3.2304 & 1.5953 \\
\hline
\end{tabular}

relatively coarse models. Of course, model $b$ results in a better solution compared to model $\mathrm{c}$ due to the fact that model $\mathrm{c}$ is coarser than model $\mathrm{b}$. Table 2 shows the values of the recovery error for the three models.

Also, it is worth mentioning that the computational time of solving both inverse problems, (26) and (27), is the same since the modeling error term is computed in advance. The total computational time required for solving an inverse problem based on the Bayesian approximation error approach comprises two parts: the computational time required for representing the modeling error stochastically and the computational time required for minimizing (27). However, the computational time required for solving an inverse problem based on the traditional Bayesian approach is only the time required for minimizing (27).

The computational time required for representing the modeling error stochastically is $\left(Z \times\left(t_{f}+t_{c}\right)\right)$, with $t_{f}$ and $t_{c}$ being the time required for solving one forward problem in the fine or coarse model, respectively. On the other side, the used Levenberg-Marquardt method for minimizing (26) or (27) solves the forward problem $p+1$ times, where $p$ is the number of unknowns. Hence, one iteration takes $\left((p+1) \times t_{f}\right)$. Consequently, the total CPU time of the traditional inverse problem is $\left(\left(4 \times t_{f}\right) \times\right.$ no of iterations $)$. So the total computational time required for solving an inverse problem based on the traditional Bayesian approach is $\left(t_{\operatorname{Trad}}=\left(4 \times t_{f}\right) \times\right.$ no of iterations $)$. And the total computational time required for solving an inverse problem based on the Bayesian approximation error approach is $\left(t_{\text {Compensated }}=Z \times\left(t_{f}+t_{c}\right)+\left(4 \times t_{f}\right) \times\right.$ no of iterations $)$.
Table 3. The computational time, in min, required for solving the traditional Bayesian approach (26) and Bayesian approximation error approach (27) for models b and c compared to the time required for solving (26) for model a, assuming $Z=100$ and the number of iterations $=100$.

\begin{tabular}{lllll}
\hline & & & $\begin{array}{l}\text { Traditional } \\
\text { Bayesian }\end{array}$ & $\begin{array}{l}\text { Bayesian approximation } \\
\text { error }\end{array}$ \\
\hline Model a & 10 & - & $4 \times 10^{3}$ & - \\
Model b & - & 1 & $0.4 \times 10^{3}$ & $1.5 \times 10^{3}$ \\
Model c & - & 0.1667 & 66.68 & $1.08 \times 10^{3}$ \\
\hline
\end{tabular}

Table 3 shows the computational time, in min, required for solving (26) and (27) for models $b$ and c compared to the time required for solving (26) for model a, assuming $Z=100$ and the number of iterations equal to 100 . It is clear from table 3 that the time consumed for solving the Bayesian approximation error approach for model $b$ is appreciably less than the traditional Bayesian approach for model a, i.e. more than 2.5 times less.

The results presented in this section validate 'experimentally' the proposed approach.

\section{Conclusion}

In this paper, we proposed a Bayesian statistical approach for compensating the modeling error originating from the simplification in the computer model. The main concept of the approach is to take into account not only the measurement noise but also the uncertainty arising from the imperfection of the applied forward model. The prime advantage of 
the proposed approach is the reduction of the error of reconstruction when using a coarse model, and consequently, numerically less demanding forward solvers. We applied the methodology for the magnetic material characterization of two electromagnetic devices, i.e. a switched reluctance motor and an EI core inductor. Both numerical and experimental results are presented in this paper showing a large reduction in the modeling error within a relatively small computational time. Moreover, we introduced a new parameter to assess the degree of the coarseness of a coarse model. This model coarseness criterion is used for determining a priori the usefulness of the applicability of the Bayesian approximation error approach. Future research will be focused on investigating the applicability of the method toward higher dimensional inverse problems and onto the modeling error reduction when neglecting the magnetic anisotropy effects in different electromagnetic devices, e.g. power transformers.

\section{Acknowledgments}

This work was supported by project GOA07/GOA/006 funded by the Ghent university. GC is a postdoctoral researcher of the 'Fund of Scientific Research Flanders' (FWO).

\section{Q2 References}

[1] Antonelli E, Cardelli E and Faba A 2005 Epstein frame: How and when it can be really representative about the magnetic behavior of laminated magnetic steels IEEE Trans. Magn. 41 1516-9

[2] Miyagi D, Miki K, Nakano M, Otome D and Takahashi N 2010 Influence of compressive stress on magnetic properties of laminated electrical steel sheets IEEE Trans. Magn. 46 318-21

[3] Abdallh A, Sergeant P, Crevecoeur G, Vandenbossche L, Dupré L and Sablik M J 2009 Magnetic material identification in geometries with non-uniform electromagnetic fields using global and local magnetic measurements IEEE Trans. Magn. 52 4157-60

[4] Emery A and Nenarokomovz A 1998 Optimal experiment design Meas. Sci. Technol. 9 864-76

[5] Abdallh A and Dupré L 2010 Local magnetic measurements in magnetic circuits with highly non-uniform electromagnetic fields Meas. Sci. Technol. 21045109

[6] Abdallh A, Crevecoeur G and Dupré L 2010 Optimal needle placement for the accurate magnetic material quantification based on uncertainty analysis in the inverse approach Meas. Sci. Technol. 21115703

[7] Abdallh A, Crevecoeur G and Dupré L 2011 Selection of measurement modality for magnetic material characterization of an electromagnetic device using stochastic uncertainty analysis IEEE Trans. Magn. 47 4564-73
[8] Crevecoeur G, Abdallh A, Couckuyt I, Dupré L and Dhaene T 2011 Two-level refined direct optimization scheme using intermediate surrogate models for electromagnetic optimization of a switched reluctance motor Eng. Comput. at press

[9] Nissinen A, Heikkinen L and Kaipio J 2008 The Bayesian approximation error approach for electrical impedance tomography—experimental results Meas. Sci. Technol. 19015501

[10] Tarvainen T, Kolehmainen V, Pulkkinen A, Vauhkonen M, Schweiger M, Arridge S and Kaipio J 2010 An approximation error approach for compensating for modelling errors between the radiative transfer equation and the diffusion approximation in diffuse optical tomography Inverse Problems 26015005

[11] Nissinen A, Heikkinen L, Kolehmainen V and Kaipio J 2009 Compensation of errors due to discretization, domain truncation and unknown contact impedances in electrical impedance tomography Meas. Sci. Technol. 20105504

[12] Nissinen A, Kolehmainen V and Kaipio J 2011 Compensation of modelling errors due to unknown domain boundary in electrical impedance tomography IEEE Trans. Med. Imaging 30 231-42

[13] Abdallh A, Sergeant P, Crevecoeur G and Dupré L 2010 An inverse approach for magnetic material characterization of an EI core electromagnetic inductor IEEE Trans. Magn. 46 622-5

[14] Bandler J, Cheng Q, Dakroury S, Mohamed A, Bakr M, Madsen K and Søndergaard J 2004 Space mapping: state of the art IEEE Trans. Microw. Theory Tech. 52 337-61

[15] Abdallh A, Crevecoeur G, Sergeant P and Dupré L 2011 Magnetic material identification of a switched reluctance motor Int. J. Appl. Electromagn. Mech. 37 35-49

[16] Nissinen A, Kolehmainen V and Kaipio J 2010 Compensation of errors due to incorrect model geometry in electrical impedance tomography J. Phys.: Conf. Ser. 224012050

[17] Kaipio J and Somersalo E 2005 Statistical and Computational Inverse Problems (Applied Mathematical Sciences vol 160) (New York: Springer)

[18] Emery A, Valenti E and Bardot D 2007 Using Bayesian inference for parameter estimation when the system response and experimental conditions are measured with error and some variables are considered as nuisance variables Meas. Sci. Technol. 18 19-29

[19] Ostovic V 1989 Dynamics of Saturated Electric Machines (New York: Springer)

[20] Marquardt D 1963 An algorithm for least-squares estimation of nonlinear parameters SIAM J. Appl. Math. 11 431-41

[21] Viana F, Venter G and Balabanov V 2010 An algorithm for fast optimal Latin hypercube design of experiments Int. J. Numer. Methods Eng. 82 135-56

[22] Cale J, Sudhoff S and Turner J 2006 An improved magnetic characterization method for highly permeable materials IEEE Trans. Magn. 42 1974-81

[23] Shan S and Wang G 2010 Survey of modeling and optimization strategies to solve high-dimensional design problems with computationally-expensive black-box functions Struct. Multidiscip. Optim. 41 219-41 


\section{QUERIES}

Page 1

Q1

Author: Please be aware that the color figures in this article will only appear in color in the Web version. If you require color in the printed journal and have not previously arranged it, please contact the Production Editor now.

\section{Page 12}

Q2

Author: Please check the details for any journal references that do not have a blue link as they may contain some incorrect information. Pale purple links are used for references to arXiv e-prints.

Q3

Author: Please update reference [8] with complete details (volume number, page range). 